HNO 2011 · 59:223-223

DOI 10.1007/s00106-011-2292-1

Online publiziert: 10. März 2011

(c) Springer-Verlag 2011

P.K. Plinkert • M. Praetorius

HNO-Klinik, Universitätsklinikum Heidelberg

\title{
Preisträger in der HNO und QR-Codes
}

Den Preis der Arbeitsgemeinschaft Olfaktologie und Gustologie (BurghartFörderpreis Chemosensorik) hat Herr Dr. Thomas Bitter von der HNO-Klinik der Universität Jena erhalten. Er hat sich mit der voxelbasierten Morphometrie beschäftigt und diese auf die Messung von Volumenänderungen der grauen Hirnsubstanz bei Anosmikern angewandt. Insbesondere diese 3 Beiträge unterstreichen die breite Aufstellung unseres Fachgebiets, die auch die Sinnesphysiologie genuin umfasst.

Die Arbeitsgemeinschaft Plastische, rekonstruktive und ästhetische KopfHals-Chirurgie (APKO) hat ihren Preis Herrn Dr. Achim Feucht aus der HNOKlinik der Universität Ulm verliehen. Er hat sich mit dem freien mikrochirurgischen Transfer von in vitro gezüchteten Knorpelkonstrukten und deren Neovaskularisation beschäftigt.

\section{จ Als Neuerung bieten wir die Möglichkeit, Videos mittels QR-Codes abzurufen}

Als eine wesentliche Neuerung und Unterstützung der wissenschaftlichen Inhalte wollen wir in dieser Zeitschrift ab sofort, dort wo dies sinnvoll ist, auch die Möglichkeit bieten, Videos mittels QR-Codes („quick response codes") abrufbar zu machen. Den Anfang macht ein erläuterndes Video zu dem Beitrag von PD Dr. Christian Simon aus der HNO-Klinik der Universität Heidelberg zur transoralen Roboterchirurgie in diesem Heft. Dem Trend zu den Smartphones und den mit ihnen einhergehenden mobilen Online-Möglichkeiten wollen wir Rechnung tragen.
Wir wünschen Ihnen eine spannende Lektüre mit den Ergebnissen der Preisträger!

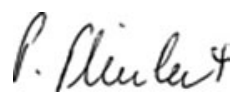

Prof. Dr. Dr. h.c. Peter K. Plinkert

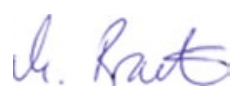

PD Dr. Mark Praetorius

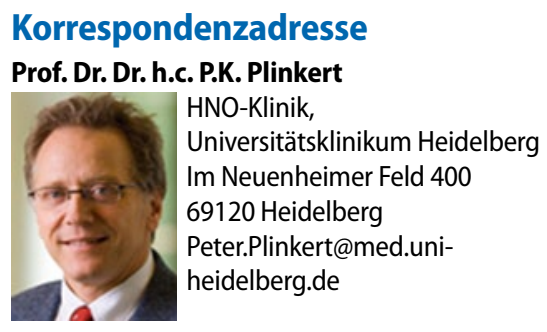

PD Dr. M. Praetorius

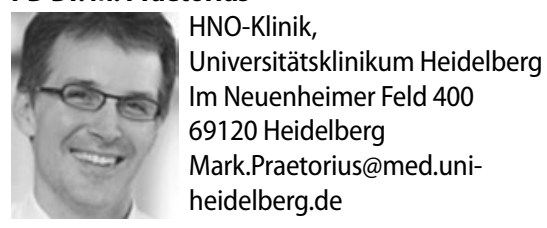

